\title{
An Economic Decision Support System based on Fuzzy Cognitive Maps with Evolutionary Learning Algorithm
}

\author{
Katarzyna Poczęta, Łukasz Kubuś, Alexander Yastrebov \\ Kielce University of Technology \\ al. Tysiąclecia Państwa Polskiego 7 \\ 25-314 Kielce, Poland \\ Email: \{k.piotrowska,lkubus,jastri\}@tu.kielce.pl
}

\author{
Elpiniki I. Papageorgiou \\ Technological Educational Institute (T.E.I.) of Central Greece \\ 3rd Km Old National Road Lamia-Athens \\ Lamia 35100, Greece \\ Email: epapageorgiou@teiste.gr
}

\begin{abstract}
Fuzzy cognitive map (FCM) is a universal tool for modeling dynamic decision support systems. It can be constructed by the experts or learned based on data. FCM models learned from data are denser than those created by experts. We developed an evolutionary learning approach for fuzzy cognitive maps based on density and system performance indicators. It allows to select only the most significant connections between concepts and receive the structure more similar to the FCMs initialized by experts. This paper is devoted to the application of the developed approach to model an economic decision support system. The learning and testing process was accomplished with the use of historical data.
\end{abstract}

\section{INTRODUCTION}

$\mathbf{F}$ UZZY cognitive map (FCM) is a directed graph for representing causal reasoning [9]. Nodes are variable concepts important for the analyzed problem and links are causal connections. FCM can be used for modeling decision support systems [12], [25], [26], e.g. for political decision making [4], artificial emotions forecasting [20] or prediction of work of complex systems [24].

Fuzzy cognitive map can be initialized based on expert knowledge. Experts select the concepts of the map and determine the weights of the connections between them (connection matrix). The second way to build the FCM model are learning algorithms [15]. Supervised [8], [18] and evolutionary learning algorithms [1], [3], [6], [12], [16], [22], [27] allow to determine the connection matrix based on historical data.

The resulting matrices are much denser than the models created by the humans. The density of the FCMs developed by experts is usually in the range of $30 \%-40 \%$ [23]. They choose only the most significant connections between concepts. FCMs with the smaller density are more readable for humans. Developing the learning algorithms that allow to build models in a manner more similar to human reasoning is an important part of research related to the fuzzy cognitive maps. A sparse real-coded genetic algorithm was proposed to utilize the density of the FCM model [23]. In [17], [19], a structure optimization genetic algorithm was introduces. A multi-objective evolutionary algorithm for learning maps with varying densities was analyzed in [7].
In [11], we propose a new evolutionary learning approach for fuzzy cognitive maps learning based on density and system performance indicators (SPI) analysis. It allows to obtain some compromise between data, density and significance of the connections. System performance indicators introduced by Borisov [5] and Silov [21] allow to analyze reliability of the FCM model and determine the total (direct and indirect) influence between concepts. The evaluation of the candidate FCMs is based on data error, density and the total influence between concepts. The obtained results based on the synthetic and reallife data generated from the reference matrices provided by experts proved that the developed approach allows to receive structure of the FCM model more similar to the reference object keeping the similar level of data error.

This paper is devoted to the use of fuzzy cognitive map with the developed evolutionary algorithm based on SPI in modeling economic decision support system. The aim of the analysis is to approximate the historical data and determine the influence between harmonized indexes of consumer prices for Poland [28]. The comparison of the developed approach with the standard one based on data error and the approach based on density was done. The learning process was accomplished with the use of Elite Genetic Algorithm (EGA) and Individually Directional Evolutionary Algorithm (IDEA) [10].

Section II presents fuzzy cognitive maps. Section III describes system performance indicators. The developed evolutionary algorithm for fuzzy cognitive maps learning is described in Section IV. In Section V, the results of experiments based on historical economic data are presented. Section VI contains the conclusion of the paper.

\section{FuZZy COGNITIVE MAPS}

Fuzzy cognitive map is a directed graph in the form [9]:

$$
<X, W>
$$

where $X=\left[X_{1}, \ldots, X_{n}\right]^{T}$ is the set of the concepts, $W$ is the connection matrix describing weights of the connections, $w_{j, i}$ is the weight of the direct influence between the $j$-th concept and the $i$-th concept, taking on the values from the 
range $[-1,1]$. A positive weight of the connection $w_{j, i}$ means $X_{j}$ causally increases $X_{i}$. A negative weight of the connection $w_{j, i}$ means $X_{j}$ causally decreases $X_{i}$.

Fuzzy cognitive map can be used for modeling behavior of dynamic systems. The state of the FCM model is determined by the values of the concepts at the $t$-th iteration. The simulation of the FCM behavior requires an initial state vector. Next, the values of the concepts can be calculated according to the selected dynamic model. Simulations show the effect of the changes in the states of the map and can be used in a what-if analysis [2]. In the paper a popular dynamic model was used [22]:

$$
X_{i}(t+1)=F\left(\sum_{j=1, j \neq i}^{n} w_{j, i} \cdot X_{j}(t)\right)
$$

where $X_{i}(t)$ is the value of the $i$-th concept at the $t$-th iteration, $i=1,2, \ldots, n, n$ is the number of concepts, $t$ is discreet time, $t=0,1,2, \ldots, T$. Transformation function $F(x)$ normalizes values of the concepts to a proper range. A logistic function is most often used [3], [22], [23]:

$$
F(x)=\frac{1}{1+e^{-c x}}
$$

where $c$ is a parameter, $c>0$.

\section{SYSTEM PERFORMANCE INDICATORS}

System performance indicators allow to evaluate the structure of the FCM model e.g. by analysis the influence between concepts. Connection matrix $W$ describes direct influence between concepts. The total influence $p_{j, i}$ between concepts means the maximum direct or indirect influence between concepts. To determine this system performance indicator the total causal effect path between concepts can be calculated [5], [21].

Algorithm for determining SPI contains the following steps [5], [21]:

1) First, connection matrix $W$ with positive and negative direct relationships between concepts passes to matrix $R$ size $2 n \times 2 n$ with positive relationships as follows:

$$
\begin{aligned}
& \text { if } w_{j, i}>0 \\
& \text { then } r_{2 j-1,2 i-1}=w_{j, i}, r_{2 j, 2 i}=w_{j, i} \\
& \text { if } w_{j, i}<0 \\
& \text { then } r_{2 j-1,2 i}=-w_{j, i}, r_{2 j, 2 i-1}=-w_{j, i}
\end{aligned}
$$

2) Next, operation of transitive closure of the matrix $R$ is used:

$$
R^{*}=R \vee R^{2} \vee R^{3} \vee \ldots
$$

where $\vee$ means maximum operation, $R^{k}$ is calculated in accordance with the max-product composition:

$$
R^{k}=R^{k-1} \circ R
$$

3) Elements of the matrix $R^{*}$ are transformed into matrix $V$ as follows:

$$
\begin{aligned}
& v_{j, i}=\max \left(r_{2 j-1,2 i-1}, r_{2 j, 2 i}\right) \\
& v_{j, i}^{\prime}=-\max \left(r_{2 j-1,2 i}, r_{2 j-1,2 i}\right)
\end{aligned}
$$

4) On the basis of the matrix $V$ the total (direct and indirect) influence between the $j$-th concept and the $i$-th concept is calculated:

$$
\begin{gathered}
\text { for } v_{j, i} \neq v_{j, i}^{\prime} \\
p_{j, i}=\operatorname{sign}\left(v_{j, i}+v_{j, i}^{\prime}\right) \max \left(\left|v_{j, i}\right|,\left|v_{j, i}^{\prime}\right|\right)
\end{gathered}
$$

where $p_{j, i}$ takes value in the range of $[-1,1]$.

5) The total influence between concepts can be used to determine other system performance indicators, for example the impact of the $j$-th concept on the system (9) and the impact of the system on the $i$-th concept (10):

$$
\begin{aligned}
& \overrightarrow{P_{j}}=\frac{1}{n} \sum_{i=1}^{n} p_{j, i} \\
& \overleftarrow{P_{i}}=\frac{1}{n} \sum_{j=1}^{n} p_{j, i}
\end{aligned}
$$

\section{EVOlutionary LEARNing Algorithm BASED ON SPI}

Evolutionary algorithms (like RCGA or EGA) can be applied to learn the FCM model (determine the weights of the connections between concepts) based on the available historical data. Each individual in the population is represented by a floating-point vector [22]:

$$
W^{\prime}=\left[w_{1,2}, \ldots, w_{1, n}, w_{2,1}, w_{2,3}, \ldots, w_{2, n}, \ldots, w_{n, n-1}\right]^{T}
$$

where $w_{j, i}$ is the weight of the connection between the $j$-th and the $i$-th concept.

The individuals are decoded into the candidate FCMs and the response of every model are calculated based on the learning initial state vectors. The aim of the standard evolutionary algorithms for fuzzy cognitive maps learning is to minimize a total difference between the normalized historical data and the model response (data error), described as follows [3], [22]:

$$
T E=\sum_{p=1}^{P} \sum_{t=1}^{T} \sum_{i=1}^{n}\left|Z_{i}^{p}(t)-X_{i}^{p}(t)\right|
$$

where $t=0,1,2, \ldots, T, T$ is the learning record length, $Z_{i}^{p}(t)$ is the reference value of the $i$-th concept at iteration $t$ for the $p$-th learning record, $X_{i}^{p}(t)$ is the value of the $i$-th concept at iteration $t$ of the candidate FCM started from the $p$-th initial state vector, $p=1,2, \ldots, P, P$ is the number of the learning records.

Fuzzy cognitive maps learned with the use of methods based on data error properly perform the task of the input data approximation. However, the resulting connection matrices are denser than those initialized by experts [23]. Density of the FCM model can be expressed as a ratio of the number of non-zero weights and number of all possible non-zero weights according to the formula:

$$
\text { density }=\frac{w_{\text {non }- \text { zero }}}{n^{2}-n}
$$

where $w_{\text {non-zero }}$ is the number of non-zero weights $w_{j, i}, n$ is the number of the concepts. 
To solve the problem of density the extensions of the standard evolutionary algorithms based on density analysis were proposed:

- sparse real-coded genetic algorithm [23],

- structure optimization genetic algorithm [17], [19],

- multi-objective evolutionary algorithm for learning FCM models with varying densities [7].

In [11], we introduced a new evolutionary approach for fuzzy cognitive maps learning that allow to determine the weights of the connections in the way similar to human reasoning. It is based on the analysis of data error, density and total influence between concepts in order to select only the most significant connections. Experiments performed with the use of synthetic and real-life data (generated from the reference FCMs) confirm that the resulting models are more similar to the reference systems.

The developed algorithm has the following objectives [11]:

- to minimize the data error (12),

- to minimize the FCM model density (13),

- to maximize the ratio of the number of significant total influences between concepts to the number of all possible influences described as follows:

$$
\text { PChRR }=\frac{p_{\text {relevant }}}{n^{2}}
$$

where $p_{\text {relevant }}$ is the number of total influences with the absolute value greater than $0.5\left(\left|p_{j, i}\right|>0.5\right)$.

To obtain some compromise between data error, density and significance of the influences between concepts, weighting method for determine the objective function was used [14]:

$$
\begin{gathered}
\text { Error }=a_{1} \cdot T E+a_{2} \cdot \text { density } \cdot \text { TE }+ \\
+a_{3} \cdot(1-P C h R R) \cdot T E
\end{gathered}
$$

where $a_{1}, a_{2}, a_{3}$ are parameters that meet the following condition:

$$
\sum_{i=1}^{3} a_{i}=1
$$

Density and total influence objectives are multiplied by the total error in order to lie in the same range.

Each candidate FCM is evaluated with the use of the following fitness function:

$$
\text { fitness }(\text { Error })=- \text { Error }
$$

The developed approach consists of the following steps [11]:

STEP 1. Initialize random population.

Random initial population is generated and evaluated with the use of the fitness function. Each generated individual has density greater or equal to $20 \%$ and lower or equal to $50 \%$.

STEP 2. Check stop condition.

If the number of iterations is greater than iteration $_{\max }$ then stop the learning process.

STEP 3. Use evolutionary algorithm to generate new population.

In the simulation analysis of the proposed approach Elite Genetic Algorithm and Individually Directed Evolutionary Algorithm were used [10].
STEP 4. Analyze population.

The values from $[-0.05,0.05]$ are rounded down to 0 as suggested in [22]. The total influence between concepts $p_{j, i}$ is calculated. The weight value $w_{j, i}$ is rounded down to 0 if the value of $p_{j, i}$ is in the interval $[-0.2,0.2]$. Additionally, density is checked. Go to STEP 2.

STEP 5. Choose the best individual and test it.

\section{A. Elite Genetic Algorithm}

Elite Genetic Algorithm (EGA) [13] uses floating-point encoding as Real-Coded Genetic Algorithm (RCGA) and elite strategy. In the first step, random initial population is generated and evaluated. Next, temporary population $T^{t}$ is created from current base population $P^{t}$ by proportionate selection (roulette-wheel selection) with dynamic linear scaling of fitness function. Individuals of temporary population $T^{t}$ are modified by Uniform Crossover operator and Non-Uniform Mutation operator [13]. The Uniform Crossover uses a fixed mixing ratio between two parents called exchange probability and usually it is equal to 0.5. Non-Uniform Mutation (NUM) operator keeps the population from stagnating in the early stages and decreases the range of mutation in later stages of evolution. In the last step, the temporary population $T^{t}$ becomes new base population $P^{t+1}$ after evaluation of the temporary population individuals.

\section{B. Individually Directed Evolutionary Algorithm}

Individually Directional Evolutionary Algorithm (IDEA) [10] uses floating-point encoding as EGA expanded by the additional mutation direction vector $(D V)$. The $D V$ is used by the mutation operation and correction of mutation direction process in post-selection stage. Random initial population is generated and evaluated as for EGA. Next, the roulette-wheel selection with dynamic linear scaling of fitness function is used to create temporary population $T^{t}$. Next, Directional NonUniform Mutation operator (DNUM) is used to create the second population $T^{\prime} t$ based on the temporary population $T^{t}$ [10]. Individuals of temporary population $T^{\prime} t$ are evaluated and the next base population is created with the use of post-selection. The individual of temporary population $T^{t}$ is compared to the corresponding individual from temporary population $T^{\prime}$. Better individual is selected for the next base population. If the mutated individual is worse than the corresponding individual, the corresponding element of directional vector of the primary individual is corrected (correction of mutation direction).

\section{EXPERIMENTS}

To analyze the performance of the developed evolutionary algorithm for fuzzy cognitive maps learning historical data were used [28]. The aim of the experiments is to build the economic decision support systems that allows to approximate the input data and analyze the influence between harmonized indexes of consumer prices for Poland. The comparative analysis of the developed approach with the standard one based on data error and the approach based on density was done. 


\section{A. Evaluation criteria}

To evaluate the resulting FCM models, two criteria were calculated:

1) similarity between the input learning data and the data generated by the FCM candidate [22]:

$$
\text { initial }_{\text {error }}=\frac{1}{P \cdot T \cdot n} \sum_{t=1}^{T} \sum_{p=1}^{P} \sum_{i=1}^{n}\left|Z_{i}^{p}(t)-X_{i}^{p}(t)\right|
$$

where $t=0,1,2, \ldots, T, T$ is the learning record length, $Z_{i}^{p}(t)$ is the reference value of the $i$-th concept at iteration $t$ for the $p$-th learning record, $X_{i}^{p}(t)$ is the value of the $i$-th concept at iteration $t$ of the candidate FCM started from the $p$-th initial state vector, $p=1,2, \ldots, P$, $P$ is the number of the learning records.

2) generalization capabilities of the candidate FCM (similarity between the input testing data and the data generated by the FCM candidate) [22]:

$$
\text { behavior }_{\text {error }}=\frac{1}{P \cdot T \cdot n} \sum_{p=1}^{P} \sum_{t=1}^{T} \sum_{i=1}^{n}\left|Z_{i}^{p}(t)-X_{i}^{p}(t)\right|
$$

where $t=0,1,2, \ldots, T, T$ is the testing record length, $Z_{i}^{p}(t)$ is the reference value of the $i$-th concept at iteration $t$ for the $p$-th testing record, $X_{i}^{p}(t)$ is the value of the $i$-th concept at iteration $t$ of the candidate FCM started from the $p$-th initial state vector, $p=1,2, \ldots, P$, $P$ is the number of the testing records.

\section{B. Dataset}

The analyzed dataset contains monthly harmonized indexes of consumer prices for Poland collected in the period from January, 2011 to December, 2015 [28]:

- $X_{1}$ - date,

- $X_{2}$ - overall Index Excluding Alcohol and Tobacco for Poland,

- $X_{3}$ - all Items Excluding Administered Prices for Poland,

- $X_{4}$ - all Items Excluding Fully Administered Prices for Poland,

- $X_{5}$ - all Items Excluding Mainly Administered Prices for Poland

- $X_{6}$ - overall Index Excluding Energy for Poland,

- $X_{7}$ - overall Index Excluding Education, Health, and Social Protection for Poland,

- $X_{8}$ - overall Index Excluding Energy, Food, Alcohol, and Tobacco for Poland,

- $X_{9}$ - overall Index Excluding Energy and Seasonal Food for Poland,

- $X_{10}$ - overall Index Excluding Liquid Fuels and Lubricants for Personal Transport Equipment for Poland

- $X_{11}$ - overall Index Excluding Energy and Unprocessed Food for Poland,

- $X_{12}$ - overall Index Excluding Housing, Water, Electricity, Gas, and Other Fuels for Poland,

- $X_{13}$ - overall Index Excluding Seasonal Food for Poland,
- $X_{14}$ - overall Index Excluding Tobacco for Poland.

Historical data were normalized according to the following equation:

$$
f(x)=\frac{x-\min }{\max -\min },
$$

where $x$ is an input numeric value, $\min$ is the minimum of the dataset, $\max$ is the maximum of the dataset.

Data were divided into sequences of 10 consecutive records. 3 sequences were used as the learning records, and 3 sequences were used as the testing records.

\section{Learning parameters}

The following parameters were used for the EGA algorithm:

- selection method: roulette wheel selection with linear scaling

- recombination method: uniform crossover,

- crossover probability: 0.75 ,

- mutation method: non-uniform mutation,

- mutation probability: 0.02,

- population size: 10 ,

- number of elite individuals: 2 ,

- maximum number of iterations: 500,

The following parameters were used for the IDEA algorithm:

- selection method: roulette wheel selection with linear scaling

- mutation method: directed non-uniform mutation,

- mutation probability: $\frac{1}{n^{2}-n}$

- population size: 10 ,

- maximum number of iterations: 500,

The standard approach based on data error was realized for the objective function described by the equation (12). Table I shows the parameters of the objective function (15) for the approach based on density. Table II shows the parameters of the objective function (15) for the approach based on density and SPI. 10 experiments were performed for every set of the learning parameters and the average values (Avg) and standard deviations (Std) were calculated.

TABLE I

PARAMETERS OF THE OBJECTIVE FUNCTION FOR THE APPROACH BASED ON DENSITY ANALYSIS

\begin{tabular}{cccc}
\hline \hline L.p. & $a_{1}$ & $a_{2}$ & $a_{3}$ \\
\hline 1 & 0.9 & 0.1 & 0 \\
2 & 0.8 & 0.2 & 0 \\
3 & 0.7 & 0.3 & 0 \\
4 & 0.6 & 0.4 & 0 \\
5 & 0.5 & 0.5 & 0 \\
\hline
\end{tabular}

\section{Results}

Figure 1 shows an exemplary economic decision support system based on fuzzy cognitive map with the developed evolutionary algorithm. Table III summarizes the results of the experiments with historical data obtained for the standard approach (STD), the approach based on density (DEN) and the 
TABLE II

PARAMETERS OF THE OBJECTIVE FUNCTION FOR THE PROPOSED APPROACH

\begin{tabular}{cccc}
\hline \hline L.p. & $a_{1}$ & $a_{2}$ & $a_{3}$ \\
\hline 1 & 0.8 & 0.1 & 0.1 \\
2 & 0.1 & 0.1 & 0.8 \\
3 & 0.7 & 0 & 0.3 \\
4 & 0.4 & 0.3 & 0.3 \\
5 & 0.3 & 0.4 & 0.3 \\
6 & 0.3 & 0.3 & 0.4 \\
7 & 0.6 & 0 & 0.4 \\
8 & 0.33 & 0.33 & 0.33 \\
\hline \hline
\end{tabular}

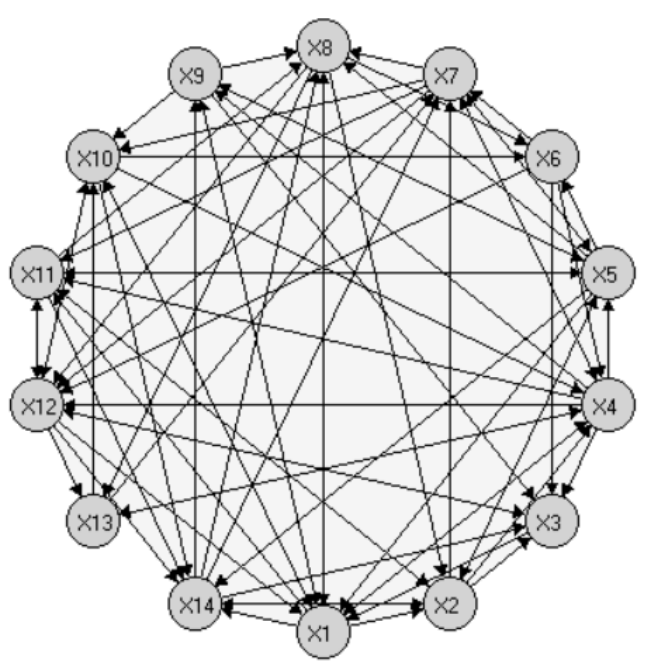

Fig. 1. Fuzzy cognitive map for economic decision support system

proposed approach (SPI). The obtained results show that the developed approach allows to approximate the economic data with satisfactory accuracy similar to the standard approach and the approach based on density. Figure 2 shows the sample results of testing of the resulting economic decision support systems.

The best solutions for the analyzed approaches are presented below. Table IV presents the resulting connection matrix obtained for the standard approach. Connection matrix for the approach based on density is shown in Table V. Table VI presents the connection matrix obtained for the proposed approach. The connection matrix for the standard approach has density $99 \%$. The density for the second connection matrix (Table V) is equal $42 \%$. The connection matrix for the evolutionary algorithm based on SPI has the lower density $40 \%$. The FCM models obtained for the developed approach and method based on density are more readable and easier to interpret than the map obtained with the standard learning algorithm based only on data error.

For further analysis, impact of the $j$-th concept on the system (9) and impact of the system on the $i$-th concept (10) were calculated and presented in Table VII. The indicators obtained for the algorithm based on SPI have the highest absolute values. The developed approach allows to keep only
TABLE III

EXPERIMENTAL RESULTS WITH HISTORICAL DATA

\begin{tabular}{|c|c|c|c|c|}
\hline Approach & Method & Parameters & $\begin{array}{c}\text { initial }_{\text {error }} \\
\mathrm{Avg} \pm \mathrm{Std}\end{array}$ & $\begin{array}{c}\text { behavior }_{\text {error }} \\
\text { Avg } \pm \text { Std }\end{array}$ \\
\hline STD & IDEA & & $0.0427 \pm 0.0001$ & $0.0411 \pm 0.0001$ \\
\hline STD & EGA & & $0.0428 \pm 0.0001$ & $0.0411 \pm 0.0001$ \\
\hline \multirow[t]{5}{*}{ DEN } & IDEA & 1 & $0.0427 \pm 0.0001$ & $0.0411 \pm 0.0001$ \\
\hline & & 2 & $0.0428 \pm 0.0001$ & $0.0411 \pm 0.0001$ \\
\hline & & 3 & $0.0428 \pm 0.0001$ & $0.0411 \pm 0.0001$ \\
\hline & & 4 & $0.0640 \pm 0.0671$ & $0.0628 \pm 0.0686$ \\
\hline & & 5 & $0.0428 \pm 0.0001$ & $0.0411 \pm 0.0001$ \\
\hline \multirow[t]{5}{*}{ DEN } & EGA & 1 & $0.0433 \pm 0.0002$ & $0.0417 \pm 0.0003$ \\
\hline & & 2 & $0.0436 \pm 0.0004$ & $0.0419 \pm 0.0005$ \\
\hline & & 3 & $0.0435 \pm 0.0003$ & $0.0418 \pm 0.0003$ \\
\hline & & 4 & $0.0436 \pm 0.0004$ & $0.0420 \pm 0.0004$ \\
\hline & & 5 & $0.0435 \pm 0.0002$ & $0.0418 \pm 0.0002$ \\
\hline \multirow[t]{8}{*}{ SPI } & IDEA & 1 & $0.0427 \pm 0.0001$ & $0.0411 \pm 0.0001$ \\
\hline & & 2 & $0.0445 \pm 0.0053$ & $0.0429 \pm 0.0054$ \\
\hline & & 3 & $0.0490 \pm 0.0133$ & $0.0475 \pm 0.0137$ \\
\hline & & 4 & $0.0444 \pm 0.0050$ & $0.0427 \pm 0.0052$ \\
\hline & & 5 & $0.0428 \pm 0.0001$ & $0.0411 \pm 0.0001$ \\
\hline & & 6 & $0.0469 \pm 0.0071$ & $0.0453 \pm 0.0073$ \\
\hline & & 7 & $0.0538 \pm 0.0142$ & $0.0524 \pm 0.0145$ \\
\hline & & 8 & $0.0428 \pm 0.0001$ & $0.0411 \pm 0.0001$ \\
\hline \multirow[t]{8}{*}{ SPI } & EGA & 1 & $0.0568 \pm 0.0227$ & $0.0555 \pm 0.0233$ \\
\hline & & 2 & $0.0468 \pm 0.0069$ & $0.0452 \pm 0.0070$ \\
\hline & & 3 & $0.0596 \pm 0.0237$ & $0.0584 \pm 0.0244$ \\
\hline & & 4 & $0.0567 \pm 0.0197$ & $0.0553 \pm 0.0203$ \\
\hline & & 5 & $0.0516 \pm 0.0120$ & $0.0501 \pm 0.0123$ \\
\hline & & 6 & $0.0592 \pm 0.0251$ & $0.0579 \pm 0.0257$ \\
\hline & & 7 & $0.0482 \pm 0.0078$ & $0.0466 \pm 0.0080$ \\
\hline & & 8 & $0.0608 \pm 0.0240$ & $0.0595 \pm 0.0246$ \\
\hline
\end{tabular}

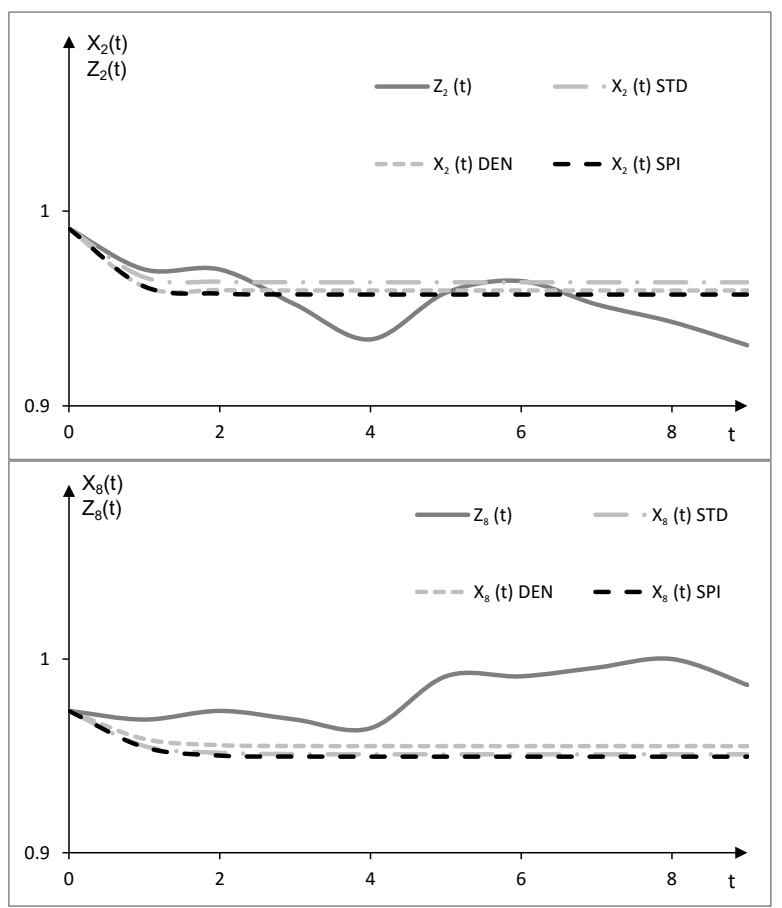

Fig. 2. Sample results of testing

the most significant connections between concepts and receive the structure for which all the concepts have a major impact 
TABLE IV

CONNECTION MATRIX FOR THE FCM LEARNED WITH THE USE OF THE STANDARD APPROACH

\begin{tabular}{|c|c|c|c|c|c|c|c|c|c|c|c|c|c|c|}
\hline$w_{j, i}$ & $X_{1}$ & $X_{2}$ & $X_{3}$ & $X_{4}$ & $X_{5}$ & $X_{6}$ & $X_{7}$ & $X_{8}$ & $X_{9}$ & $X_{10}$ & $X_{11}$ & $X_{12}$ & $X_{13}$ & $X_{14}$ \\
\hline$X_{1}$ & 0 & 0.9 & -0.15 & 0.98 & -0.21 & 0.65 & 0.39 & 0.22 & 0.64 & -0.17 & 0.56 & 0.42 & 0.82 & 0.21 \\
\hline$X_{2}$ & 0.97 & 0 & 0.34 & 0.18 & 0.92 & 0.28 & -0.33 & -0.4 & -0.11 & -0.74 & 0.69 & -0.69 & 0.07 & -0.35 \\
\hline$X_{3}$ & 0.64 & -0.88 & 0 & -0.43 & 0.44 & 0.83 & 0.47 & 0.07 & -1 & 0.2 & 0.56 & -0.13 & 0.36 & 0.66 \\
\hline$X_{4}$ & -1 & 0.05 & 0.05 & 0 & -0.89 & -0.46 & 0.33 & -0.14 & 0.99 & 0.91 & 0.04 & 0.08 & -0.01 & 0.8 \\
\hline$X_{5}$ & 0.52 & 0.55 & 0.32 & 0.54 & 0 & 0.94 & 0.41 & 0.9 & 0.38 & 0.04 & -0.67 & 0.86 & 0.71 & -0.95 \\
\hline$X_{6}$ & -0.66 & 0.77 & 0.21 & 0.62 & -0.15 & 0 & 0.4 & 0.22 & 0.99 & 0.12 & 0.24 & 0.71 & 0.62 & 0.49 \\
\hline$X_{7}$ & 0.57 & -0.73 & -0.28 & 0.48 & -0.61 & -0.13 & 0 & -0.24 & -0.14 & -0.14 & -0.9 & 0.7 & 0.61 & -0.24 \\
\hline$X_{8}$ & -0.15 & 0.08 & 0.31 & -0.1 & 0.76 & 0.88 & -0.09 & 0 & 0.56 & 0.4 & 0 & -0.2 & 0.57 & 0.73 \\
\hline$X_{9}$ & 0.42 & 0.03 & 0.8 & -0.36 & 0.9 & 0.01 & -1 & 0.85 & 0 & 0.94 & -0.17 & 0.13 & -0.66 & 0.13 \\
\hline$X_{10}$ & -0.36 & -0.4 & -0.31 & -0.62 & 0.05 & -0.34 & 0.94 & 0.51 & 0.78 & 0 & 0.97 & 0.27 & 0.88 & 0.71 \\
\hline$X_{11}$ & 0.89 & 0.87 & 0.75 & 0.21 & -0.13 & -1 & -0.17 & -0.37 & 0.87 & 0.52 & 0 & -0.18 & -0.61 & 0.43 \\
\hline$X_{12}$ & -0.05 & -0.31 & -0.41 & -0.07 & -0.34 & 0.97 & 0.64 & -0.08 & -0.46 & 0.94 & 0.89 & 0 & 0.03 & 0.52 \\
\hline$X_{13}$ & -0.89 & 0.99 & -0.39 & 0.42 & 0.93 & 0.53 & 0.96 & -0.09 & -0.25 & 0.08 & -0.67 & 0.66 & 0 & -0.55 \\
\hline$X_{14}$ & -0.07 & 0.58 & 0.97 & 0.88 & 0.59 & -0.57 & -0.42 & 0.72 & -0.52 & -0.42 & 0.88 & -0.34 & -0.72 & 0 \\
\hline
\end{tabular}

on the entire system and system has the significant impact on the concepts.

\section{Vi. Conclusion}

The paper presents the application of fuzzy cognitive maps with the developed evolutionary algorithm for fuzzy cognitive maps learning to model economic decision support system. The presented approach is based on data error, density and system performance indicators analysis. The resulting FCM models are readable and easier to interpret. The proposed approach allows to keep only the most significant connections between concepts and receive fuzzy cognitive map for which all the harmonized indexes of consumer prices have a significant impact on the entire system and system has the significant impact on the indexes, keeping satisfactory accuracy of modeling of economic data.

\section{REFERENCES}

[1] G. Acampora, W. Pedrycz and A. Vitiello, "A Competent Memetic Algorithm for Learning Fuzzy Cognitive Maps," IEEE Transactions on Fuzzy Systems, vol. 23, no. 6, pp. 2397-2411, 2015 , http://dx.doi.org/10.1109/TFUZZ.2015.2426311.

[2] J. Aguilar, "A Survey about Fuzzy Cognitive Maps Papers," International Journal of Computational Cognition,vol. 3 (2), pp. 27-33, 2005.

[3] S. Ahmadi, N. Forouzideh, S. Alizadeh, E. I. Papageorgiou, "Learning Fuzzy Cognitive Maps using Imperialist Competitive Algorithm," Neural Computing and Applications, vol. 26(6), pp. 1333-1354, 2015 http://dx.doi.org/10.1007/s00521-014-1797-4.

[4] A. S. Andreou, N. H. Mateou, and G. A. Zombanakis, "Soft Computing for Crisis Management and Political Decision Making: The Use of Genetically Evolved Fuzzy Cognitive Maps," Soft Computing Journal, Vol.9, Issue 3, pp. 194-210, 2005, http://dx.doi.org/10.1007/s00500-0040344-0.

[5] V. V. Borisov, V. V. Kruglov, and A. C. Fedulov, Fuzzy Models and Networks, Publishing house Telekom, Moscow, 2004 (in Russian).

[6] A. Buruzs, M. F. Hatwagner, R. C. Pozna, and L. T. Koczy, "Advanced learning of fuzzy cognitive maps of waste management by bacterial algorithm," IFSA World Congress and NAFIPS Annual Meeting (IFSA/NAFIPS), pp. 890-895, 2013, http://dx.doi.org/10.1109/IFSANAFIPS.2013.6608518.

[7] Y. Chi and J. Liu, "Learning of Fuzzy Cognitive Maps with Varying Densities using Multi-objective Evolutionary Algorithms," IEEE Transactions on Fuzzy Systems, pp. 71-81, 2015, http://dx.doi.org/10.1109/TFUZZ.2015.2426314.

[8] A. Jastriebow and K. Poczęta, "Analysis of multi-step algorithms for cognitive maps learning," Bulletin of the Polish Academy of Sciences Technical Sciences, vol. 62, Issue 4, pp. 735-741, 2014, http://dx.doi.org/10.2478/bpasts-2014-0079.
[9] B. Kosko, "Fuzzy cognitive maps," International Journal of Man-Machine Studies, vol. 24, no.1, pp. 65-75, 1986, http://dx.doi.org/10.1016/S0020-7373(86)80040-2.

[10] Ł. Kubuś, "Individually Directional Evolutionary Algorithm for Solving Global Optimization Problems - Comparative Study," International Journal of Intelligent Systems and Applications (IJISA), Vol. 7, No. 9, 2015, str. 12-19.

[11] Ł. Kubuś, K. Poczęta, and A. Yastrebov, "A New Learning Approach for Fuzzy Cognitive Maps based on System Performance Indicators," 2016 IEEE International Conference on Fuzzy Systems, Vancouver, Canada, pp. 1-7, 2016.

[12] N. H. Mateou and A. S. Andreou, "A Framework for Developing Intelligent Decision Support Systems Using Evolutionary Fuzzy Cognitive Maps," Journal of Intelligent and Fuzzy Systems, Vol. 19, Number 2, pp. 171-150, 2008.

[13] Z. Michalewicz, Genetic algorithms + data structures = evolution programs, Springer-Verlag, New York, 1996.

[14] K. M. Miettinem, Nonlinear Multiobjective Optimization, Kluwer Academic Publishers, Boston, 1999.

[15] E. I. Papageorgiou, "Learning Algorithms for Fuzzy Cognitive Maps A Review Study." IEEE Transactions on Systems, Man, and Cybernetics - Part C: Applications and Reviews, vol. 42, no. 2, pp. 150-163, 2012, http://dx.doi.org/10.1109/TSMCC.2011.2138694

[16] E. I. Papageorgiou, K. E. Parsopoulos, C. D. Stylios, P. P. Groumpos, and M. N. Vrahtis, "Fuzzy Cognitive Maps Learning Using Particle Swarm Optimization," Journal of Intelligent Information Systems, 25:1, pp. 95-121, 2005, http://dx.doi.org/10.1007/s10844-005-0864-9.

[17] E.I. Papageorgiou, K. Poczeta, and C. Laspidou, "Application of Fuzzy Cognitive Maps to water demand prediction," Fuzzy Systems (FUZZ IEEE), 2015 IEEE International Conference on, Istanbul, pp. 1-8, 2015 http://dx.doi.org/10.1109/FUZZ-IEEE.2015.7337973.

[18] K. Poczęta and A. Yastrebov, "Analysis of Fuzzy Cognitive Maps with Multi-Step Learning Algorithms in Valuation of OwnerOccupied Homes," 2014 IEEE International Conference on Fuzzy Systems (FUZZ-IEEE), Beijing, China, pp.1029-1035, 2014 http://dx.doi.org/10.1109/FUZZ-IEEE.2014.6891587.

[19] K. Poczęta, A. Yastrebov, and E. I. Papageorgiou, "Learning Fuzzy Cognitive Maps using Structure Optimization Genetic Algorithm," 2015 Federated Conference on Computer Science and Information Systems (FedCSIS), Lodz, Poland, pp. 547-554, 2015, http://dx.doi.org/10.15439/2015F296.

[20] J. L. Salmeron, "Fuzzy cognitive maps for artificial emotions forecasting," Applied Soft Computing, vol. 12, pp. 3704-3710, 2012, http://dx.doi.org/10.1016/j.asoc.2012.01.015.

[21] V. B. Silov, Strategic decision-making in a fuzzy environment. Moscow: INPRO-RES, 1995 (in Russian).

[22] W. Stach, L. Kurgan, W. Pedrycz, and M. Reformat, "Genetic learning of fuzzy cognitive maps," Fuzzy Sets and Systems, vol. 153, no. 3, pp. 371-401, 2005, http://dx.doi.org/10.1016/j.fss.2005.01.009.

[23] W. Stach, W. Pedrycz, and L. A. Kurgan, "Learning of fuzzy cognitive maps using density estimate," IEEE Trans. on Systems, Man, and Cybernetics, Part B, vol. 42(3), pp. 900-912, 2012, http://dx.doi.org/10.1109/TSMCB.2011.2182646. 
TABLE V

CONNECTION MATRIX FOR THE FCM LEARNED WITH THE USE OF THE APPROACH BASED ON DENSITY

\begin{tabular}{|c|c|c|c|c|c|c|c|c|c|c|c|c|c|c|}
\hline$w_{j, i}$ & $X_{1}$ & $X_{2}$ & $X_{3}$ & $X_{4}$ & $X_{5}$ & $X_{6}$ & $X_{7}$ & $X_{8}$ & $X_{9}$ & $X_{10}$ & $X_{11}$ & $X_{12}$ & $X_{13}$ & $X_{14}$ \\
\hline$X_{1}$ & 0 & 0.54 & 0 & 0.47 & 0 & 0 & 0.28 & 0 & 0 & 0 & 0.98 & 0 & 0.99 & 0 \\
\hline$X_{2}$ & -0.78 & 0 & 0 & 0 & 0 & -0.21 & 0 & 0 & 0 & 0 & 0 & 0.32 & 0 & 0.89 \\
\hline$X_{3}$ & 0.56 & 0 & 0 & 0.77 & 0.9 & 0.53 & -0.23 & 0.8 & 0 & 0.58 & 0.77 & 0.11 & -0.32 & 0.36 \\
\hline$X_{4}$ & 0 & 0 & 0 & 0 & 0 & 0 & 0 & 0 & 0 & 0 & 0 & 0 & 0 & -0.97 \\
\hline$X_{5}$ & 0.5 & 0 & 0.91 & 0.79 & 0 & 0 & 0.56 & 0 & 0 & 0.81 & 0 & -0.74 & 0.88 & 0 \\
\hline$X_{6}$ & 0 & -0.49 & 0 & 0 & 0 & 0 & 0 & 0.91 & 0.29 & 0 & 0.69 & 0 & 0 & 0.79 \\
\hline$X_{7}$ & 0 & 1 & 0.9 & 0 & 0 & 0 & 0 & 0.07 & 0 & 0.33 & 0 & 0 & 0 & 0 \\
\hline$X_{8}$ & 0.77 & 0 & 0 & 0.34 & 0.46 & 0 & 0 & 0 & 0.47 & 0.55 & 0 & 0 & 0 & 0.59 \\
\hline$X_{9}$ & 0 & 0 & 0.86 & 0 & 0.91 & 0.99 & 0.66 & 0.97 & 0 & 0 & 0 & 0.71 & 0.62 & 0.92 \\
\hline$X_{10}$ & 0.35 & 0 & 0 & 0.88 & 0 & -0.66 & 0.99 & 0 & 0 & 0 & 0 & 0 & 0 & 0 \\
\hline$X_{11}$ & -0.9 & 0 & 0 & -0.14 & 0 & 0.94 & 0.27 & 0 & 0.88 & 0 & 0 & 0.96 & 0.12 & 0 \\
\hline$X_{12}$ & 0 & 0.61 & 0 & 0 & 0 & 0.99 & 0 & 0 & 1 & 0.37 & 0 & 0 & 0 & 0 \\
\hline$X_{13}$ & -0.27 & 0.87 & -0.25 & -0.87 & 0 & 0 & 0 & 0 & 0 & 0 & 0 & 0 & 0 & 0 \\
\hline$X_{14}$ & 0.6 & 0 & 0 & 0.45 & 0 & -0.11 & 0 & -0.57 & 0 & 0 & 0 & 0.87 & 0.42 & 0 \\
\hline
\end{tabular}

TABLE VI

CONNECTION MATRIX FOR THE FCM LEARNED WITH THE USE OF THE PROPOSED APPROACH

\begin{tabular}{|c|c|c|c|c|c|c|c|c|c|c|c|c|c|c|}
\hline$w_{j, i}$ & $X_{1}$ & $X_{2}$ & $X_{3}$ & $X_{4}$ & $X_{5}$ & $X_{6}$ & $X_{7}$ & $X_{8}$ & $X_{9}$ & $X_{10}$ & $X_{11}$ & $X_{12}$ & $X_{13}$ & $X_{14}$ \\
\hline$X_{1}$ & 0 & 0.8 & 0 & 0.24 & 0 & 0 & 0.66 & 0.72 & -0.89 & 0.56 & 0 & 0 & -0.79 & 0 \\
\hline$X_{2}$ & 0 & 0 & 0 & 0.7 & -0.22 & -0.31 & 0 & 0 & 0 & 0 & 0.98 & 0 & 0 & 0 \\
\hline$X_{3}$ & 0 & 0 & 0 & 0 & 0.87 & 0 & 0 & -0.34 & 0 & 0 & 0 & 0.2 & 0 & 0.99 \\
\hline$X_{4}$ & -0.36 & 0.86 & 0 & 0 & 0 & 0.59 & 0 & 0 & 0 & 0.65 & 0.58 & 0 & 0 & -0.31 \\
\hline$X_{5}$ & 0 & 0 & 0 & 0 & 0 & 0.99 & -0.11 & 0 & 0 & 0 & 0 & 1 & 0 & 0 \\
\hline$X_{6}$ & 0 & 0 & 0.77 & 0 & 0 & 0 & 0.73 & 0 & 0 & 0.93 & 0 & 0 & 0.49 & -0.83 \\
\hline$X_{7}$ & 0 & 0 & 0 & 0 & -0.66 & 0 & 0 & 0 & 0.63 & 0 & 0 & 0 & 1 & 0.67 \\
\hline$X_{8}$ & 0 & 0 & 0 & 0 & 0 & 0 & 0 & 0 & 0.96 & 0 & 0 & 0.81 & 0 & 0 \\
\hline$X_{9}$ & 0.81 & 0 & 0 & 0 & 0.87 & 0 & 0 & -0.11 & 0 & 0 & 0.71 & 0 & 0.87 & -0.2 \\
\hline$X_{10}$ & 0 & 0 & 0 & 0 & 0.93 & 0 & 0 & 0 & 0 & 0 & 0 & 0.49 & 0.2 & 0.58 \\
\hline$X_{11}$ & -0.33 & 0.79 & 0.48 & 0.77 & 0 & 0.65 & 0.36 & 0.38 & 0.52 & 0.62 & 0 & 0 & 0.98 & 0.89 \\
\hline$X_{12}$ & 0 & 0 & 1 & 0.98 & 0 & 0.9 & 0.23 & -0.32 & 0.39 & 0 & 0 & 0 & -0.31 & 0 \\
\hline$X_{13}$ & 0 & 0 & 0 & 0 & 0.47 & 0 & 0 & 0.94 & 0.91 & 0 & 0.08 & -0.26 & 0 & 0.79 \\
\hline$X_{14}$ & 0.7 & 0 & 0 & 0 & 0 & -0.35 & 0.72 & 0.97 & 0 & 0 & 0 & 0 & 0 & 0 \\
\hline
\end{tabular}

TABLE VII

EXPERIMENTAL RESULTS WITH HISTORICAL DATA

\begin{tabular}{ccccccc}
\hline \hline \multirow{2}{*}{ Concept } & \multicolumn{2}{c}{ STD } & \multicolumn{2}{c}{ DEN } & \multicolumn{2}{c}{ SPI } \\
& $\vec{P}_{j}$ & $\overleftarrow{P_{i}}$ & $\vec{P}_{j}$ & $\overleftarrow{P_{i}}$ & $\vec{P}_{j}$ & $\overleftarrow{P_{i}}$ \\
\hline$X_{1}$ & 0.25 & 0.22 & 0.62 & -0.22 & -0.44 & 0.62 \\
$X_{2}$ & 0.50 & 0.36 & 0.30 & 0.47 & 0.80 & 0.76 \\
$X_{3}$ & -0.37 & -0.34 & 0.56 & 0.67 & 0.84 & 0.73 \\
$X_{4}$ & -0.14 & 0.36 & -0.73 & 0.22 & 0.70 & 0.72 \\
$X_{5}$ & 0.36 & 0.37 & 0.26 & 0.68 & 0.90 & 0.71 \\
$X_{6}$ & 0.36 & 0.12 & 0.58 & 0.59 & 0.80 & 0.71 \\
$X_{7}$ & 0.00 & -0.26 & 0.45 & 0.58 & 0.78 & 0.65 \\
$X_{8}$ & 0.43 & 0.35 & 0.59 & 0.71 & 0.79 & 0.78 \\
$X_{9}$ & 0.22 & 0.26 & 0.82 & 0.58 & 0.81 & 0.75 \\
$X_{10}$ & 0.13 & 0.38 & 0.49 & 0.56 & 0.85 & 0.67 \\
$X_{11}$ & -0.13 & 0.25 & 0.37 & 0.11 & 0.80 & 0.76 \\
$X_{12}$ & 0.49 & 0.33 & 0.83 & 0.57 & 0.88 & 0.72 \\
$X_{13}$ & 0.52 & 0.34 & 0.29 & 0.13 & 0.77 & 0.73 \\
$X_{14}$ & -0.23 & -0.36 & 0.74 & 0.49 & 0.79 & 0.76 \\
\hline \hline
\end{tabular}

[24] G. Słoń, "Application of Models of Relational Fuzzy Cognitive Maps for Prediction of Work of Complex Systems," Lecture Notes in Artificial Intelligence LNAI 8467, Springer-Verlag, pp. 307-318, 2014, http://dx.doi.org/10.1007/978-3-319-07173-2_27.

[25] G. Słoń, "The Use of Fuzzy Numbers in the Process of Designing Relational Fuzzy Cognitive Maps", Lecture Notes in Artificial Intelligence LNAI 7894/Part 1, Springer-Verlag, pp. 376-387, 2013, http://dx.doi.org/10.1007/978-3-642-38658-9_34.

[26] G. Słoń and A. Yastrebov, "Optimization and Adaptation of Dynamic Models of Fuzzy Relational Cognitive Maps," in: S.O. Kuznetsov et al. (Eds.) RSFDGrC 2011, Lecture Notes in Artificial Intelligence 6743, Springer-Verlag, Heidelberg, pp. 95-102, 2011, http://dx.doi.org/10.1007/978-3-642-21881-1_17.

[27] E. Yesil and L. Urbas, "Big bang: big crunch learning method for fuzzy cognitive maps," World Acad. Sci. Eng. Technol., vol. 71, pp. 815-8124, 2010 .

[28] https://research.stlouisfed.org/fred2/downloaddata/ [20.04.2016] 